\section{Our experts highlight the most important research articles across the spectrum of topics relevant to the field of hepatic oncology}

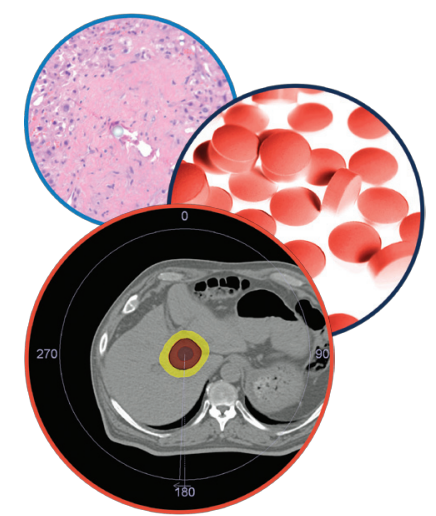

Hepatic Oncology

Expert panel: Geraldine O'Sullivan Coyne, Genitourinary Malignancies Branch, Medical Oncology Service, Center for Cancer Research, National Cancer Institute, Bethesda, MD, USA; Tim F Greten, Gastrointestinal Malignancy Section, Thoracic \& GI-Oncology Branch, Center for Cancer Research, National Cancer Institute, Bethesda, MD, USA

Choi J, Shim JH, Shin YM, Kim KM, Lim YS, Lee HC. Clinical significance of the best response during repeated transarterial chemoembolization in the treatment of hepatocellular carcinoma. J. Hepatol. 60(6), 1212-1218 (2014).

The authors compared the clinical impact of a 'best observed response' following repeated transarterial chemoembolization (TACE) sessions to a on 'best initial response' in 322 patients with intermediate-stage hepatocellular carcinoma (HCC) and preserved liver function; with $\geq 1$ liver lesion, $\geq 1 \mathrm{~cm}$ in size. They also evaluated the factors relevant to the decision for repeated TACE sessions prior to determining a 'best' response. Responses were measured using mRECIST and EASL and survival analysis was performed depending on the type of response obtained. After one TACE course, $51.2 \%$ of patients achieved an objective response (complete response or partial response) by EASL, and $53 \%$ by mRECIST. With a median of two TACE sessions (range 1-6), responders increased to $75.3 \%$ by EASL and to $83.7 \%$ by mRECIST. Multivariate COX analysis demonstrated that an objective response by both criteria was the best prognostic factor for overall survival (OS), with hazard ratios (HRs) that were lower than for those of initial responders (HR 0.45 by EASL and 0.24 by mRECIST, $\mathrm{p}<0.001$ for both; versus HR of 0.69 and $0.71, \mathrm{p}=0.05$ in first responders). The size of the largest and second largest tumors were the parameters positively correlated with the number of TACE sessions required to achieve the best response $(\mathrm{p}<0.05)$. The 'best response' during serial TACE rather than the initial response better predicted the survival of this patient population.

Fuks D, Cauchy F, Fusco G, Paradis V, Durand F, Belghiti J. Preoperative fineneedle aspiration tumor biopsy does not affect the oncologic course of patients with transplantable HCC. J. Hepatol. 61(3), 589-593 (2014).

This study evaluated whether preoperative fine-needle aspiration biopsy (PFNAB) of HCC patients compromises outcomes post liver transplantation (LT) using an intent-totreat analysis. This single-institution study evaluated 309 patients, of which 26\% of patients were PFNAB + and 74\% were PFNAB-. Both groups (PFNAB + and PFNAB-) were similar demographically, with comparable rates of lesions as per the Milan criteria (81\% vs $79 \%$ respectively, $\mathrm{p}=0.67$ ) and waiting-list times (7 vs 6.9 months respectively, $\mathrm{p}=0.891$ ). Pathological analysis in the 278 (90\%) patients that were transplanted showed that $11(4 \%)$ patients had non-HCC lesions, of which 10 were amongst the PFNAB- patient group. Median follow-up was 34 months (range 12-135). Recurrence after LT was noted in 25 (9\%) patients with no difference in either group (9.3 vs $8.9 \%, p=0.904$ ), and parietal recurrence was noted in one $\mathrm{PFNAB}+$ patient and in two PFNAB- patients after radiofrequency ablation $(p=0.797)$. The authors carried out 1, 3 and 5 year OS analysis which was not significantly different between PFNAB + or PFNAB-patients $(89,69$ and $60 \%$ vs 85,67 and
Future
Medicine 
$61 \%$, respectively, $\mathrm{p}=0.601$ ), and recommend that it is possibly to carry out PFNAB without affecting the oncological outcome to prevent unnecessary LT.

\section{Yau T, Tang VY, Yao TJ, Fan ST, Lo \\ CM, Poon RT. Development of Hong \\ Kong liver cancer staging system with treatment stratification for patients with hepatocellular carcinoma. Gastroenterology 146(7), 1691-1700 (2014).}

This paper details the development of a new prognostic classification criterion for hepatocellular carcinoma (HCC) in Asian patients. Data on 3856 patients with HCC and hepatitis B (HepB) was collected from 1995 to 2008 from a single institution. This prognostic staging system and treatment guidelines were constructed using a 'training' set subsequently validated on a similar size cohort ('test' set), where patients were randomized in a 1:1 ratio to either set. The proposed staging system includes ECOG PS (0-4), Child-Pugh Grade (A, B or C), tumor status (early, intermediate or locally advanced as determined by size, number and presence of intrahepatic vascular invasion) and presence of extrahepatic vascular invasion/metastasis. Using Cox regression and classification as well as regression tree analysis, the authors accounted for the relative effects of each of these factors in predicting overall survival (OS) and classifying treatment decisions, constructing the definite staging system and treatment recommendations by integrating clinical judgment. The criteria distinguished specific OS times, and identified subsets of the Barcelona Clinic Liver Cancer (BCLC)-staging intermediate- and advanced-stage patients for more aggressive treatments than recommended by BCLC. BCLC-B patients reclassified as HKLC-II had a 5-year OS probability of $52.1 \%$ vs $18.7 \%$, $(\mathrm{p} \geq 0.001)$ with radical therapy compared with TACE. This new staging appears to identify patients that are suitable for more aggressive treatment than the currently employed BCLC system.
Scartozzi M, Faloppi L, Svegliati Baroni G et al. VEGF and VEGFR genotyping in the prediction of clinical outcome for HCC patients receiving sorafenib: the ALICE- 1 study. Int. J. Cancer 135(5), 1247-1256 (2014).

This paper assesses the effect of VEGF and VEGFR polymorphisms on the clinical outcome of patients treated with sorafenib, looking to define specific subgroups that may benefit from treatment. ALICE-1 (Angiogenesis LiverCancEr) is a retrospective multicenter study, with 148 patients diagnosed with HCC and receiving sorafenib at the standard dose of $400 \mathrm{mg}$ continuously were available for analysis (dose reductions were applied as clinically indicated). Tumor blocks (59 patients; $40 \%$ ) or whole blood samples (89 patients; $60 \%$ ) were tested for VEGF-A, VEGFR-C and VEFGR$1,2,3$ single nucleotide polymorphisms (SNPs) by genotyping. Univariate analysis of VEGF-A alleles $\mathrm{C}$ of $\mathrm{rs} 25648, \mathrm{~T}$ of $\mathrm{rs} 833061, \mathrm{C}$ of rs699947, C of rs2010963; VEGF-C alleles T of rs4604006, G of rs664393; VEGFR-2 alleles C of rs2071559, C of rs2305948 were significant predictors of progression-free survival and OS. At multivariate analysis, rs2010963, rs4604006 and BCLC stage resulted to be independent factors influencing progression-free survival and OS. The authors comment that as BCLC was confirmed as a prognostic factor, this validates the reproducibility of their analysis. However, the prognostic or predictive value of the identified significant SNPs is difficult to determine as prior VEGF SNP analyses have not been able to clarify the biological effect of these, and should remain hypothesis-generating at present.

Financial \& competing interests disclosure The authors have no relevant affliations or financial involvement with any organization or entity with a financial interest in or financial conflict with the subject matter or materials discussed in the manuscript. This includes employment, consultancies, honoraria, stock ownership or options, expert testimony, grants or patents received or pending, or royalties.

No writing assistance was utilized in the production of this manuscript. 